\title{
A calibrated integrating sphere setup to determine the infrared spectral radiant flux of high intensity discharge lamps
}

\author{
A J Rijke ${ }^{1}$, S Nijdam ${ }^{1}$, M Haverlag ${ }^{1,2}$, J F J Janssen ${ }^{1}$, J J A M van der Mullen ${ }^{1}$ \\ and $G M$ W Kroesen ${ }^{1}$ \\ ${ }^{1}$ Department of Applied Physics, Eindhoven University of Technology, P.O. Box 513, \\ Eindhoven, The Netherlands. \\ ${ }^{2}$ Philips Lighting, LightLabs, P.O. Box 80020, 5600 JM Eindhoven, The Netherlands \\ E-mail: a.j.rijke@tue.nl
}

\begin{abstract}
A set-up that aims to determine the infrared radiation fraction of the energy balance of high intensity discharge (HID) lamps has been designed, constructed and calibrated. It consists of a high-resolution integrating sphere that can cover a wide spectral range. New in this work is that the integrating sphere measurements can be used in the infrared part of the spectrum up to $10 \mu \mathrm{m}$ and that we have calibrated the absolute intensity in that range. No calibration standards for spectral radiant flux are readily available in the infrared. Therefore, we have used a resistive heated platinum ribbon as absolute intensity reference. As a first testcase, this new set-up was used to determine the energy balance of a Philips CDM-T 70W/830 lamp, which is a type of metal halide HID lamp.
\end{abstract}

\section{Introduction}

High intensity discharge lamps (HID lamps) are used when compact light sources are needed with high output levels and efficacies. This study deals with the experimental determination of the energy balance of HID lamps, i.e. the distribution of the total output energy of the lamp over different spectral regions is investigated. By determining and studying the energy balance of the lamp, the understanding of the energy flows inside the lamp can be improved. This can aid in the improvement of existing lamps and development of new ones.

Since a long time the determination of the energy balance of HID lamps is an important aspect of HID lamp research. Studies and publications on HID lamps efficacy research and/or energy balances include those performed by Waymouth [1], Elenbaas [2], Jack and Koedam [3], De Groot and Van Vliet [4] and Wharmby [5]. From these studies it can be concluded that infrared radiation is an important contribution to the energy balance of HID lamps. Infrared radiation, either in the form of radiation from the plasma or in the form of thermal radiation from the burner wall, can consume 50$75 \%$ of the total input power.

The ALITE-II project's [6] primary focus was the infrared radiation of HID lamps; several papers regarding the infrared radiation from HID lamps have recently been published [7-9]. In these papers the focus was mainly on the continuum radiation generated by the plasma.

As far as we know, other authors studying the infrared radiation of HID lamps have based their infrared work on side-on measurements, and/or did not measure the radiation beyond about $2.5 \mu \mathrm{m}$ 
spectrally resolved [5-7]. We will use spectrally resolved absolutely calibrated integrating sphere measurements to determine the infrared contributions to the spectrum up to $10 \mu \mathrm{m}$.

An integrating sphere is a hollow sphere with a diffuse high reflectivity coating on its inner surface. See section 2.2 for a description of the integrating sphere used in this paper. The light source is placed inside the sphere. Due to the many diffuse reflections on the inner surface, light leaving a hole in the sphere is a well-mixed measure for the total (radiant) flux of the source. However, small changes in the spectral reflectivity of the inner surface can cause large differences in the signal [10]. Therefore, this measurement system has to be carefully calibrated before absolute measurements can be performed. In the visible range this is done using standard lamps, but for the infrared this is more of an issue because existing calibration sources are either not designed for the use in the (mid-)infrared, or are only suitable for side-on measurements.

A calibration procedure has been developed to perform the calibration of the integrating sphere in the infrared. In this calibration procedure a platinum strip inside the integrating sphere is heated by means of Ohmic dissipation. The temperature profile and spectral radiant flux of the platinum strip are calculated using a model, see section 3.1. This flux is integrated over the area of the strip and this integrated flux is used as the reference signal for the calibration. The integrating sphere has been calibrated in the infrared up to $10 \mu \mathrm{m}$.

Absolutely calibrated integrating sphere measurements have been performed in the infrared on a Philips CDM-T 70W/830 HID lamp. For this lamp, the output part of the energy balance is mapped out in detail, see section 4.1. The energy in the wavelength range above $10 \mu \mathrm{m}$ is determined by assuming it is following the black body radiation curve for a constant emissivity and a temperature of $1300 \mathrm{~K}$. To correct for the radiating surface and the emissivity, this black body curve is scaled to connect to the measured infrared spectrum at $10 \mu \mathrm{m}$. The amount of visible radiation in the energy balance was acquired from integrating sphere measurements at Philips Lighting Optical Calibration Laboratory $(\mathrm{OCM})$ in Eindhoven.

The HID lamp used in this paper has an efficacy of $93 \mathrm{~lm} / \mathrm{W}$. Typical values for HID lamp efficacies are between 60 and $120 \mathrm{~lm} / \mathrm{W}$, this is almost one order of magnitude higher than the typical efficacy of an incandescent lamp. For linear fluorescent tubes, typical values are between 80 and $100 \mathrm{~lm} / \mathrm{W}$. For LED lamps, typical values are 50 to $100 \mathrm{~lm} / \mathrm{W}$ (all under operating conditions). To be able to make a comparison considering the total system, also the efficiency with which the (electronic) lamp drivers operate is important. For HID lamp drivers, typical efficiencies are in the range of 90-95\%, for linear fluorescent tubes typical values are around $90 \%$ and for LED the driver efficiencies are typically between 80 and $90 \%$.

Our setup and the details of the calibration procedure are discussed in sections 2 and 3 respectively. The infrared measurements are discussed in more detail in section 4 . The output part of the energy balance of this lamp is presented in section 4.1.

\section{Experiment}

\subsection{The energy balance}

An example of a typical representation of an energy balance is given in figure 1 (after [11]). For a more thorough discussion on energy balances see e.g. [3,4]. 


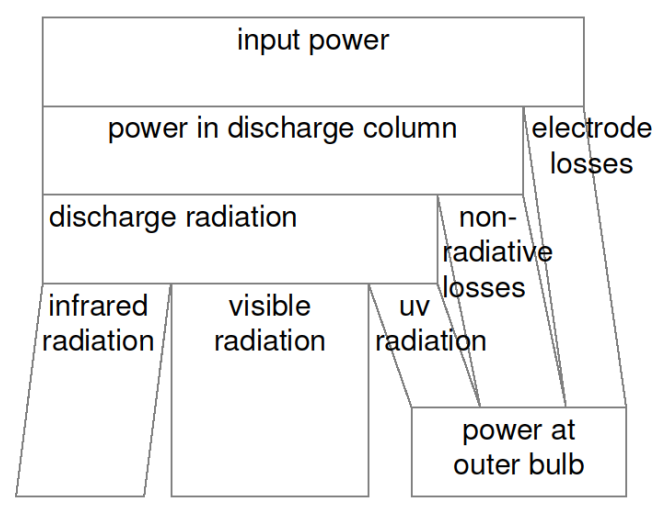

Figure 1: An example of a commonly used representation of an energy balance of a light source [11]. The power at the outer bulb is lost through conduction and convection in the surrounding gas, as well as thermal emission. An energy balance of a lamp burner operated in an evacuated environment and without the outer bulb looks slightly different. The UV radiation is no longer absorbed, and convection and conduction losses through the surrounding gas no longer appear.

For an HID lamp under normal operating conditions, the output energy consists of plasma radiation due to atomic line radiation, molecular radiation and continuum emission, as well as heat losses due to conduction, convection and thermal radiation. Since our measurements are performed on a lamp burner without outer bulb and operated in a vacuum system, for our situation the energy balance looks slightly different. The UV radiation is no longer absorbed, and convection and conduction losses through the surrounding gas no longer appear.

The outer bulb of the lamp absorbs part of the radiation. Therefore, it would obscure the information from the lamp burner. Particularly for the infrared this is an issue, as glass has a low transmission beyond 2.5 to $3.5 \mu \mathrm{m}$ depending on type and thickness. For reliable infrared measurements therefore the outer bulb has to be removed. To still be able to operate the lamp for an extended time without oxidizing its metallic components and to create a representative temperature distribution of the burner, it has to be placed in vacuum. In our setup the integrating sphere is situated inside a vacuum system. The pressure in the vacuum system during the measurements is below $5 \cdot 10^{-5} \mathrm{mbar}$.

Operating the lamp in a vacuum system has advantages and disadvantages. One advantage is that this operation excludes heat losses through convection and conduction through surrounding gas. Therefore the only loss-process besides radiation is conduction through the connecting wires. This reduces uncertainties in the final measurements. Another advantage of operating the lamp inside a vacuum system is that this reduces water vapour and $\mathrm{CO}_{2}$ absorptions in the infrared spectrum.

A disadvantage of this technique is that after removing the outer bulb, the lamp burner operates under different conditions, and this may affect lamp performance. Another disadvantage is that due to the time required to evacuate the system it takes a few hours before lamp measurements can be performed.

\subsection{The infrared integrating sphere setup}

For the experiments an integrating sphere has been designed that is dedicated to the infrared, but also suitable for the visible range. The inside of the sphere was pearl blasted and subsequently coated with aluminium to obtain a diffuse reflection in a broad spectral range. For infrared measurements gold would provide a higher reflectivity, but an aluminium coating was chosen so that measurements in the visible part of the spectrum can be performed as well.

A schematic drawing of the setup can be found in figure 2. The integrating sphere setup used for this experiment has an approximately spherical wall with an inner diameter of $28 \mathrm{~cm}$. The wall is made of 
$4 \mathrm{~mm}$ thick copper to ensure good heat conduction. Using two parabolic mirrors, the light is sent into a Bruker Vertex80 vacuum system Fourier transform spectrometer (FTIR). A baffle is placed between the exit hole and the lamp to prevent lamp light from directly entering the spectrometer. Between the integrating sphere and the FTIR the light passes a short path outside vacuum. This path is purged with nitrogen to reduce $\mathrm{CO}_{2}$ and water absorptions.

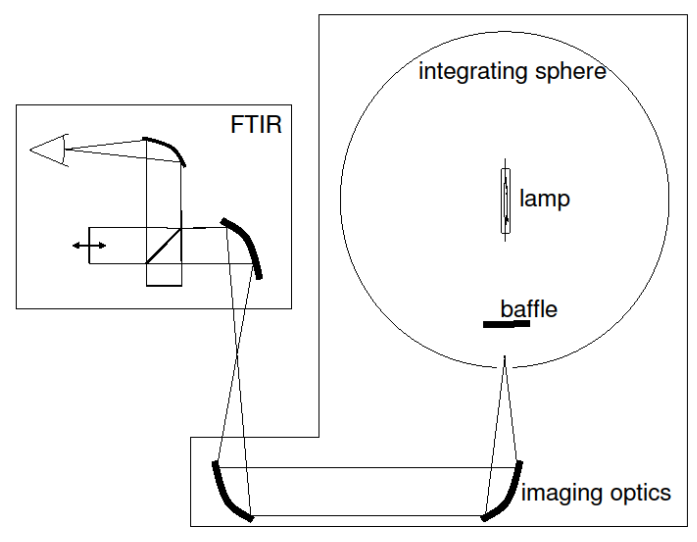

Figure 2: A schematic representation of the setup. The FTIR and the part of the setup containing the integrating sphere are evacuated. Between the integrating sphere and the FTIR the light passes a short path outside vacuum. This path is purged with nitrogen.

The ratio of the spectral radiant flux leaving the integrating sphere to the spectral radiant flux of the lamp is described by (cf. equation (10) in [10])

$$
\frac{\Phi_{\lambda \text { sphere }}}{\Phi_{\lambda \text { lamp }}}=\frac{\rho(\lambda)}{1-\rho(\lambda)} \frac{A_{\text {hole }}}{A_{\text {sphere }}} .
$$

In this equation $\Phi_{\lambda \text { sphere }}$ is the spectral radiant flux leaving the sphere, while $\Phi_{\lambda \text { lamp }}$ represents the spectral radiant flux of the lamp. The reflectivity of the inside surface of the integrating sphere is $\rho(\lambda)$. The ratio $\rho(\lambda) /(1-\rho(\lambda))$ takes the amplification of flux due to inter-reflections of the sphere into account, $A_{\text {hole }} / A_{\text {sphere }}$ is the ratio between the area of the hole and the total surface area of the integrating sphere. For this integrating sphere, the radius is about $14 \mathrm{~cm}$, and the radius of the hole is about $1 \mathrm{~cm}$. The reflectivity of the coating is estimated to be around 0.75 . For smooth aluminium the reflectivity is above 0.95 , however an oxide layer and the rough surface reduce this value. The flux leaving the integrating sphere is about $0.4 \%$ of the total lamp flux.

The integrating sphere described in [10] has the detector directly attached to the outside of the integrating sphere. In our design projecting optics, consisting of two parabolic mirrors with an aluminium surface, is used to send the radiation from the sphere into the spectrometer. Therefore, not all of the about $0.4 \%$ of the lamp flux is projected into the spectrometer. Since also effects of the lamp holder and the baffle have to be taken into account, this integrating sphere system is to complicated to calculate the calibration function purely theoretically. Therefore a calibration using a calibration source is required. This calibration will then also include contributions from the reflectivity of the projecting optics, the transmission of the windows in the optical path and the sensitivity of the detector. The calibration procedure is discussed in chapter 3.

\subsection{Comparing integrating sphere measurements to integrated side-on measurements}


With the same optics one could also measure the lamp directly instead of measuring the integrated signal. For the integrating sphere described here, the ratio of the measured signal from the integrating sphere and the measured signal from a side-on setup would be about $1 \%$. To obtain the same signal-tonoise ratio for an integrating sphere measurement as for a side-on measurement, either a much lower resolution should be selected, or a much longer integration time.

On the other hand, to accurately calculate the total emitted power of a lamp using side-on measurements, many measurements at different positions must be made and then integrated over a sphere. This requires complex rotating parts in our vacuum system. For our measurements, the integrating sphere technique is preferred.

\subsection{Temperature effects}

One of the problems encountered when measuring infrared radiation with wavelengths above $3 \mu \mathrm{m}$ is that all objects around room temperature radiate in these wavelengths as well. This will give a background signal which has to be corrected for. The intensity of a black body follows from Planck's law

$$
I(\lambda, T)=\frac{2 h c^{2}}{\lambda^{5}} \frac{1}{\exp \left(\frac{h c}{\lambda k T}\right)-1},
$$

where $h$ is Planck's constant, $c$ the speed of light, $k$ Boltzman's constant, $\lambda$ the wavelength and $T$ the temperature. To obtain the spectral radiant flux, this equation has to be integrated over the relevant solid angle and the area of the source. For non-black bodies the radiation is found by multiplying Planck's law with the spectral emissivity $\varepsilon(\lambda)$, which ranges from 0 to 1 .

In our case, especially the background radiation from the integrating sphere itself contributes significantly to the measured signal. This is because the radiating surface of the integrating sphere is much larger than that of the lamp. Furthermore the sphere will heat up by absorption of lamp radiation, so this background radiation will not be constant.

The part of the setup which is most affected by temperature effects is the baffle. Not only is the baffle the only part that is in a direct line of sight of the spectrometer, the baffle is also closer to the lamp than the rest of the sphere, and on top of that relatively loosely coupled to the rest of the sphere. Therefore, it heats up more easily than the rest of the sphere. Due to the resulting temperature difference with the rest of the sphere it also cools down much faster. To minimise temperature differences during sample- and background measurement, a temperature regulation system that runs cooling water through the baffle is operated. Heating of the surface of the baffle cannot completely be prevented, therefore a thermocouple is installed on the baffle to monitor its temperature.

Using two additional thermocouples, we measure the temperature of the integrating sphere during the lamp operation. We try to perform the background measurement at the same temperatures of the integrating sphere as during the sample measurement.

The non constant temperature puts an upper limit on the allowable integration time. To improve the signal to noise ratio, the resolution of the measurements was reduced. When comparing several background corrected measurements, we find differences in their infrared signal. The differences change direction and size when comparing different measurements, but within one measurement it is a systematic error with the following profile: the error starts increasing around $3 \mu \mathrm{m}$ and goes up to maximum $10 \%$ at $10 \mu \mathrm{m}$, wich is the end of our measurement range. Below $3 \mu \mathrm{m}$ it is negligible.

\subsection{Spectral ranges}

The Fourier transform spectrometer can be equipped with a $\mathrm{KBr}$ or a $\mathrm{CaF}_{2}$ beam splitter. For detectors we can use a room temperature DLaTGS (deuterated L-alanine doped triglycene sulphate) detector, a 
liquid nitrogen cooled MCT (mercury cadmium telluride) detector and a Si (Silicon) detector. For the experiments described in this paper, different combinations of detector and beamsplitter are used. The $\mathrm{CaF}_{2}$ beamsplitter and the $\mathrm{Si}$ detector for are used in the interval from $780 \mathrm{~nm}$ to $1.1 \mu \mathrm{m}$; the $\mathrm{CaF}_{2}$ beamsplitter and the MCT detector for the bandwidth from $1.1 \mu \mathrm{m}$ to $3.3 \mu \mathrm{m}$ and the combination of the $\mathrm{KBr}$ beamsplitter and the DLaTGS detector are used in the range from $3.3 \mu \mathrm{m}$ to $10 \mu \mathrm{m}$.

On the ports separating the integrating sphere from the Fourier transform spectrometer $\mathrm{KBr}$ windows are used. By combining the various beam splitters and detectors, this system has the possibility to cover a spectral range from $360 \mathrm{~nm}$ up to $25 \mu \mathrm{m}$. Due to the increasing uncertainty with increasing wavelength, we have limited our infrared measurement range to $10 \mu \mathrm{m}$.

\subsection{Discussion of the measurement errors}

Due to the non-constant temperature of the sphere and the baffle (see also section 2.4) a measurement error arises. This error starts contributing above $3 \mu \mathrm{m}$ and yields up to $10 \%$ at $10 \mu \mathrm{m}$, both for the calibration and the lamp measurement, resulting in a total of $20 \%$ at $10 \mu \mathrm{m}$.

For most of the spectral range, a random error of about $1 \%$ can be assumed. However, below about 1 $\mu \mathrm{m}$ the signal of the calibration source has decreased such that the random measurement error on the calibration function starts increasing. At $780 \mathrm{~nm}$ the measurement error has increased to about $20 \%$. For the lamp measurement, a random error on the calibration function becomes a systematic error. Therefore, this contribution is mentioned separately.

Over the total measurement range, a systematic error from the calculated calibration signal (4\%) is added (see section 3.2), The total relative error and which sources contribute to it is shown in figure 3 for five different wavelengths.

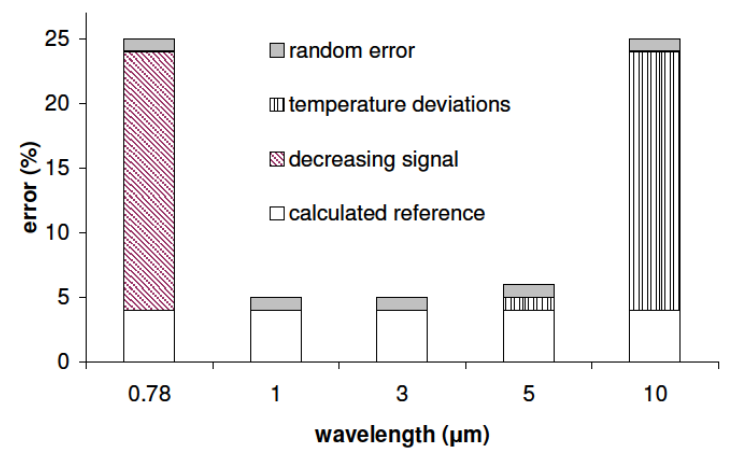

Figure 3: A bar chart indicating the error for different wavelengths and their cause. The white block is the error from the calculated emission of the calibration source. The dashed bar indicates the error due to the decreasing signal of the calibration source. The bars with the horizontal stripes indicate the error

from differences in the temperature of the integrating sphere between sample and background measurement. The gray bars indicate the random errors on the measurement.

\section{Calibration of the integrating sphere}

The key element in our calibration procedure is a resistive heated platinum strip placed inside the integrating sphere. Important properties of a calibration source are stability and reproducibility. The corrosion resistance of noble metals prevents changes in the emissivity due to oxidation. Compared to other noble metals, platinum has a good combination of a relatively high electrical resistivity with a relatively low thermal conductivity which aids in heating the strip with electric current. Our 
measurements show that the emission of the platinum strip is very stable; the emission changes with less than about $0.05 \%$ per hour.

When the platinum strip is operated inside the integrating sphere, the measured signal $S_{P_{t}}\left(\lambda, T_{P_{t}}\right)$ as a function of wavelength $\lambda$ and depending on the temperature (profile) $T_{P t}$ can be written as:

$$
\begin{aligned}
S_{P t}\left(\lambda, T_{P t}\right) & =k_{1}(\lambda) \Phi_{\lambda P t}\left(\varepsilon_{P t}(\lambda, T), T_{P t}\right) \\
& +k_{2}(\lambda) \Phi_{\lambda \text { sphere }}\left(\varepsilon_{\text {sphere }}(\lambda, T), T_{\text {sphere }}\right) \\
& +k_{3}(\lambda) R(\lambda),
\end{aligned}
$$

where $\Phi$ is the spectral radiant flux, which depends on the emissivity $\varepsilon(\lambda, T)$ and the temperature (profile) $T$. The transfer functions $k_{x}(\lambda)$ take into account effects such as the the reflectance of the integrating sphere itself $\left(k_{I}(\lambda)\right.$ and $k_{2}(\lambda)$ only), transmission of the windows, the sensitivity of the detector etc. The goal of the calibration procedure is to calculate the transfer function $k_{l}(\lambda)$. The residual signal $R(\lambda)$ is the signal from radiating sources picked up along the optical path after passing the integrating sphere.

To correct for background radiation, the result of a measurement with a cold platinum strip $\left(T_{P_{t}}=T_{e n v}\right)$ is subtracted from that of the hot platinum strip. To avoid phase correction problems in the FTspectrometer data handling, the background signal is corrected for by subtracting interferograms rather than spectra $[12,13]$. Academically speaking, the background measurement should be performed after removing the platinum strip from the sphere. However, the emission of the cold platinum strip at $10 \mu \mathrm{m}$ is rougly $1.5 \%$ of the emission of the hot strip. Hence we have a systematic error of roughly $1.5 \%$. This is much smaller than the error from the non-constant integrating sphere temperature. We measure the background radiation during cooling down of the integrating sphere. We do not have time to remove the strip from the sphere since during the removal the sphere continues to cool down. This will cause a temperature deviation of several degrees, which would result in an error in the order of $100 \%$ of the signal. We choose the smallest error, so the background measurement is done with the cold strip in the sphere.

After correcting for the background signal, the transfer function $k_{I}(\lambda)$ can be determined. It equals:

$$
\begin{aligned}
k_{1}(\lambda) & =\frac{S_{P t}\left(\lambda, T_{1}\right)-S_{P t}\left(\lambda, T_{e n v}\right)}{\Phi_{\lambda P t}\left(\varepsilon_{P t}(\lambda, T), T_{1}\right)-\Phi_{\lambda P t}\left(\varepsilon_{P t}(\lambda, T), T_{e n v}\right)} \\
& \approx \frac{S_{P t}\left(\lambda, T_{1}\right)-S_{P t}\left(\lambda, T_{e n v}\right)}{\Phi_{\lambda P t}\left(\varepsilon_{P t}(\lambda, T), T_{1}\right)}
\end{aligned}
$$

In this equation $S_{P_{t}}\left(\lambda, T_{1}\right)$ and $S_{P_{t}}\left(\lambda, T_{e n v}\right)$ are the measured signals with the hot platinum strip and the platinum strip at environmental temperature, respectively. The spectral radiant flux $\left(\Phi_{\lambda P_{t}}\right)$ of the platinum strip is calculated using the model described below.

\subsection{Model to calculate the temperature profile and the spectral radiant flux}

The model used to determine the temperature profile and the spectral radiant flux of the strip consists of two parts. The first part is a calculation of the temperature profile; the second part calculates the spectral radiant flux resulting from this temperature profile.

\subsubsection{Temperature profile calculation.}


For the temperature profile calculations, the finite control volume method is used. The main variable of interest is the temperature and 250 control volumes are used. A one dimensional temperature profile is assumed. This is justified because the length to width ratio of the strip is about 20:1 and the width to thickness ratio is about 200:1. A direct current flowing through the strip is imposed and an initial temperature profile is assumed. As is customary for the finite volume method, for the processes dependent on the main variable (temperature), a constant value (temperature) equal to the value (temperature) of the nodal point is assumed for the complete control volume. For processes dependent on the gradient of the main value (temperature), a linear interpolation is used to determine the (temperature) gradient between the nodal points. If for processes determining the interaction between two control volumes, the value of the main variable at the control volume boundary is required, this value is also determined by linear interpolation of the values at the nodal points.

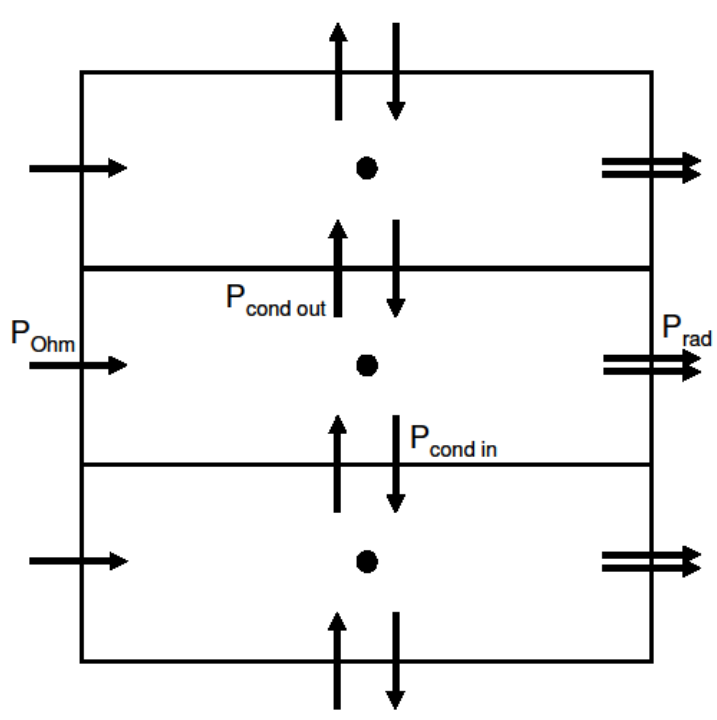

Figure 4: A schematic drawing of the power input and power loss of each control volume. The horizontal arrow pointing into the control volume indicates the power $\left(P_{O h m}\right)$ going into the control volume by Ohmic heating. In equilibrium, this Ohmic heat must equal the sum of the net power radiated away $\left(P_{\text {rad }}\right.$, indicated by the horizontal double arrows pointing out of the control volume), and the conducted heat ( $P_{c o n d}$, indicated by the arrows between the control volumes). The conducted power leaving one control volume equals the power entering the neighbouring control volume.

A schematic drawing of the power input and power loss of each control volume is given in figure 4 . For each control volume the power balance between Ohmic heating due to dissipation in the control volume, heat conduction both into and out of the control volume and radiative losses is solved. The ohmic heating, $P_{\text {ohm }}$ is calculated using Joule's first law, with the resistivity from [16]. The conducted heat $P_{\text {cond }}$ is calculated using Fourier's law of thermal conduction, with the thermal conductivity from [15]. The radiant flux $P_{\text {rad }}$ is calculated using Stefan-Boltzmann's law, with the emissivity from [14]. The equations for all the control volumes of the strip are coupled, because the heat conduction between two neighbouring cells is equal in magnitude but of opposite sign.

The excess power (or power shortage) in each iteration is used to heat up (cool down) the control volume during a time interval $\delta t$. To determine the length of $\delta t$, a Von Neumann stability analysis for heat conduction was performed. This will give us only an estimate value, since the Ohmic heating and 
radiation terms were not taken into account during this stability analysis. Therefore, an additional correction factor is added. In principle this correction factor should depend on the size of the control volume. However, in our model a factor of 0.5 works well for all settings. The equilibrium profile is assumed to have been reached when the total absolute power imbalance of all cells added together is less than 1·10-5 times the total dissipated electric power.

To validate the temperature profile, the calculated Ohmic dissipation of the strip is compared to the measured input power for four different currents (7 to $10 \mathrm{~A})$. The calculated Ohmic dissipation depends on the resistance of the strip, which in turn depends on the value for the thickness of the strip in the model. We have calculated the Ohmic dissipation for different thicknesses. The thickness which resulted in the lowest maximum deviation from the measured input power at all four currents is $28.14 \mu \mathrm{m}$. For this thickness the calculated Ohmic dissipation and the measured input power agree within $0.6 \%$ for all currents. This agrees very well with the measured thickness of the strip which is $(28 \pm 1) \mu \mathrm{m}$.

\subsubsection{Spectral radiant flux calculation}

The second part of the model calculates the spectral radiant flux of the platinum strip from the calculated temperature profile. For each control volume of the strip the spectral radiant flux is calculated using the following equation

$\Phi_{\lambda}=\pi \Delta x p(x) \varepsilon(\lambda, T) I(\lambda, T)$.

In this equation $\Delta x$ is the length of each control volume and $p(x)$ its perimeter. The spectral emissivity $\varepsilon(\lambda, T)$ is taken from Touloukian [14], which contains multiple datasets. We have chosen the dataset from Rolling et al. [17] as this is the dataset with conditions best applicable to our situation. The factor $\pi$ comes from the integration over the solid angle, assuming the radiation from the strip has a Lambertian profile, and $I(\lambda, T)$ is the black body radiation curve according to Planck's law (2).

As verification, the calculated spectral radiant flux using equation (5) is integrated over the complete spectrum and compared to the radiant flux resulting from Stefan-Boltzmann's law. Both approaches should give the same results, if the correct values for the (spectral) emissivity are used. The values agree within $5 \%$ between $1000 \mathrm{~K}$ and $1400 \mathrm{~K}$, the temperature region that causes most of the platinum strip radiation. For lower temperatures the difference goes up to $20 \%$. For the high temperatures this difference can be caused due to the radiation not having a Lambertian profile. For the lower temperatures error is more probably a result of discrepancies between the literature values. At each temperature the spectral emissivity has been multiplied by a constant correction factor to correct for the difference. This corrected spectral emissivity is then used in equation (5) to calculate the spectral radiant flux of each control volume. To obtain the spectral radiant flux of the calibration source as a whole, equation (5) is summed over all control volumes. Temperature profiles and the radiant flux profiles are shown in figure 5 for three different currents. 

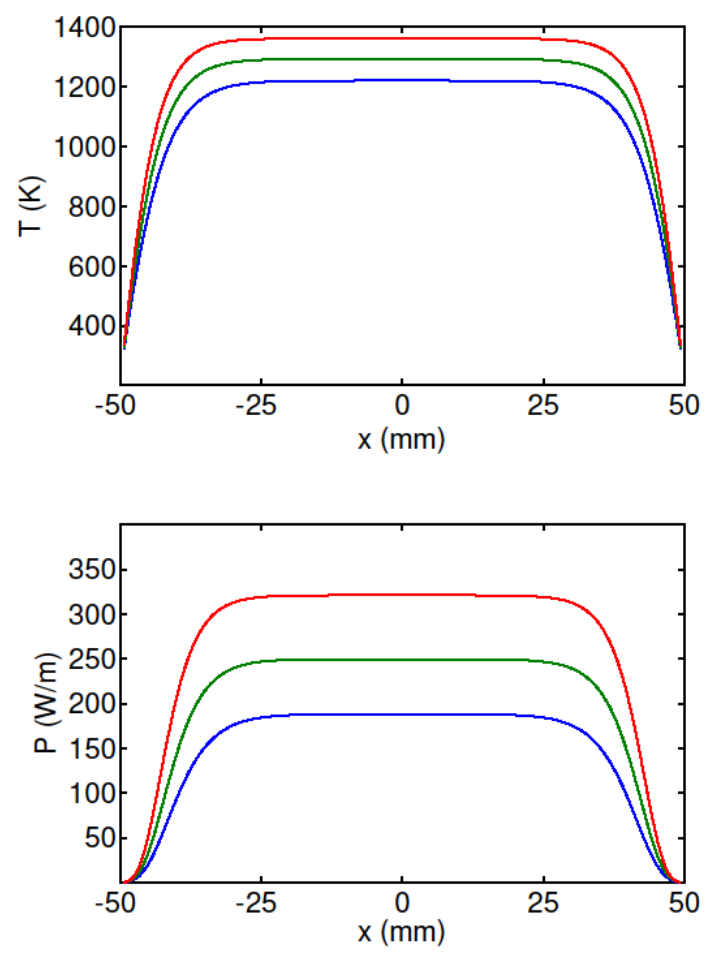

Figure 5: The temperature profile (top) of the strip and the resulting radiant flux profile (bottom) for different currents (from top to bottom 10, 9 and 8 ampere). A smooth profile with a large plateau in the centre and steep edges is found.

\subsection{Verifying the calibration function}

The total emitted power depends on the current flowing through the strip. To verify the calibration, the transfer function $k_{I}(\lambda)$, equation (4), is calculated for three different currents, and two measurements are performed for each current. The transfer function is a property of the system, and therefore does not depend on the current. The two measurements for each current yield results for $k_{l}(\lambda)$ which are within $1 \%$. When comparing two different currents, $k_{l}(\lambda)$ varies over the wavelength axis and $k_{l}(\lambda)$ deviates maximum $4 \%$ between one current and the other. This can have many different reasons: errors in the calculated temperature profile, error in the (literature) value of the resistivity of platinum (at high temperatures), error in the emissivity of platinum etc. However, at the moment we accept this $4 \%$ error.

\section{Lamp Measurement}

As a test case, infrared integrating sphere measurements have been performed on a CDM-T 70W/830 HID lamp. For the lamp a measurement procedure similar to the calibration measurements is performed. The measured signal with the lamp switched on inside the integrating sphere is:

$$
S_{\text {lamp }}(\lambda)=k_{1}(\lambda) \Phi_{\lambda \text { lamp }}+k_{2}(\lambda) \Phi_{\lambda \text { sphere }}+k_{3}(\lambda) R(\lambda)
$$

In this equation $\Phi_{\lambda \text { lamp }}$ is the spectral radiant flux of the lamp. For the rest, this equation is the same as equation (3). To correct for the background signal, the signal with the lamp switched off is subtracted. 
Again this background correction is performed using the interferograms. The spectral radiant flux of the lamp is:

$\Phi_{\lambda \text { lamp }}=\frac{S_{\text {lamp }}(\lambda)-S_{\text {lamp off }}(\lambda)}{k_{1}(\lambda)}-\Phi_{\lambda \text { lamp off }}$.

As discussed for the platinum strip calibration measurements, the background measurement is taken with the source switched off, but still inside the sphere, and subsequently neglecting the term $\Phi_{\lambda \text { lamp off }}$. Since $k_{l}(\lambda)$ has been determined previously, no unknowns are left in (7) and the spectral radiant flux emitted by the lamp can be calculated. For all the three combinations of detector and beam-splitter the measurements and calculations are performed. The resulting combined spectrum is shown in figure 6 .

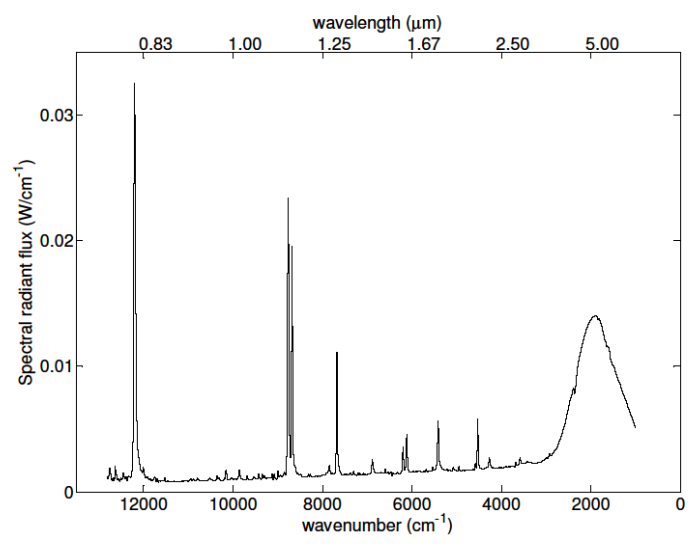

Figure 6: The absolutely calibrated infrared integrating sphere spectrum of a CDM-T 70W/830 lamp. The bulge on the right is the thermal radiation from the burner. Almost all of the strong lines in the spectrum can be attributed to $\mathrm{Na}$ emission Lines.

Measurements for the visible radiation are performed at Philips Lighting Optical Calibration Laboratory (OCM) in Eindhoven [18]. The integrating sphere measurement method has been used. Their integrating sphere was calibrated with a calibration source traceable to the National Physics Laboratory (NPL) of the UK. The power in the visible part of the spectrum of this lamp is found to be $23.2 \pm 0.7 \mathrm{~W}$.

This value has to be adapted to our situation before we can use it in the energy balance. Firstly, this spectral power is determined for a lamp power of $71.5 \mathrm{~W}$. During our infrared measurements, the lamp power was $72.3 \mathrm{~W}$. If we assume that the visible power scales linearly with total power this means the visible power during our measurement was $23.8 \pm 0.7 \mathrm{~W}$. Secondly, the Philips measurements were performed with a lamp inside its outer bulb. The transmission of the outer bulb is not equal to one. The visible radiation leaving the lamp burner therefore has to be scaled with a factor $1 / \tau(\lambda)$. Wharmby [5] has determined a value of 0.95 for the transmission of the outer bulb. Since our lamps are not the same, in our case the value could be a few percent different, but for this test case we will use 0.95 as an estimate.

\subsection{The energy balance of a CDM-T 70W/830 HID lamp}

The measured total power in the infrared spectrum shown in figure 6 is $35.3 \pm 3.0 \mathrm{~W}$. The energy in the wavelength range above $10 \mu \mathrm{m}$ is determined by assuming the spectral radiant flux is following the black body radiation curve, integrated over the solid angle, for a constant emissivity, and for a temperature of $1300 \mathrm{~K}$. To correct for the radiating surface and the emissivity, this black body curve is 
scaled to connect to the measured infrared spectrum at $10 \mu \mathrm{m}$. With this extension, an additional $2.0 \pm 0.5 \mathrm{~W}$ is found in the far infrared.

The power in the visible part of the spectrum of this lamp is found to be $25.0 \pm 0.7 \mathrm{~W}$. About $4 \%$ of the lamp power can be attributed to UV radiation [18]. For this lamp that means $2.9 \pm 0.7 \mathrm{~W}$.

Also power loss due to conduction through the connecting wires has to be taken into account. When assuming that for the lamp burner this is comparable to the conduction loss of the platinum strip, which area and temperature are comparable to that of the burner, the conduction loss is estimated to be $3.0 \mathrm{~W} \pm 1 \mathrm{~W}$. The total lamp power adds to $68.2 \pm 5.9 \mathrm{~W}$. The total electric power fed to the lamp is $72.3 \pm 0.1 \mathrm{~W}$. The two values agree fairly well; the difference is only $4.1 \mathrm{~W}$ or $5.6 \%$. The output energy balance is shown schematically in figure 7. To fill out the complete energy balance, more measurements and subsequent data processing is required, as well as lamp modelling.

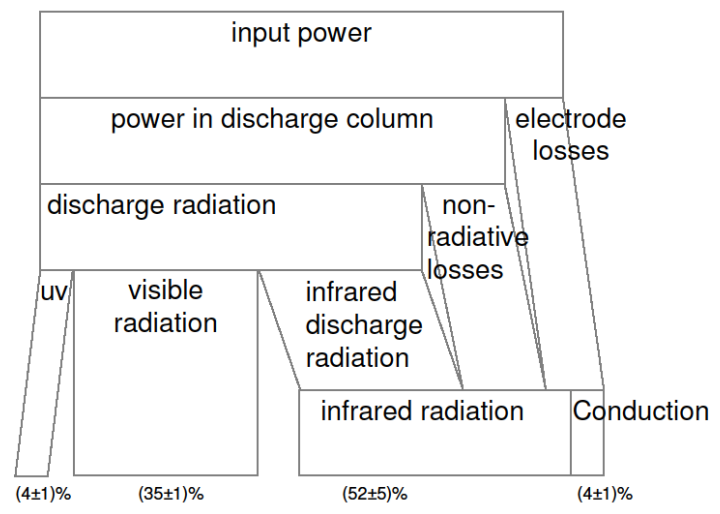

Figure 7: Energy balance of a CDM-T 70W/830 HID lamp. The burner is operated in vacuum and without outer bulb.

\section{Conclusions and outlook}

An integrating sphere setup for infrared measurements has been constructed, which is capable of performing absolutely calibrated integrated infrared measurements. As a test case, the infrared radiation from a CDM-T 70W/830 HID lamp has been determined. The total infrared power of the lamp can be determined with an accuracy of between 5 and $10 \%$. This over-all accuracy is determined by the accuracy with which the spectral radiant flux can be determined. It has been shown that a significant part of the error is due to temperature differences in the integrating sphere between the sample measurement and background measurement. In the near future we plan to further improve the accuracy of the calibration by implementing a better temperature control in the integrating sphere. Furthermore we plan to implement a dynamic switch-off experiment in order to make the distinction between IR plasma continuum radiation and radiation from the lamp wall.

\section{Acknowledgements}

Peter Weerdesteijn, Jannet van der Veen and Jos Suijker are acknowledged for their contributions to the experimental setup, and for determining the visible and the UV contributions to the energy balance.

\section{References}

[1] Waymouth J F, 1971 , 'Electric Discharge lamps', Cambridge, Massachusetts: MIT Press

[2] Elenbaas W, 1972, 'Light Sources', London: Macmillen

[3] Jack A G and Koedam M, Energy balances for some high gas pressure discharge lamps. 1974, $J$. Illum. Eng. Soc. 3 p 323-9 
[4] de Groot J and van Vliet J, 1986, The High-Pressures Sodium Lamp, Deventer: Kluwer Technishe Boeken B.V.

[5] Wharmby D O, Energy balance of high-pressure sodium discharges under controlled vapour conditions. 1984, J. Phys. D: Appl. Phys. 17 p 367-78.

[6] Gough A, Sansonetti C, Curry J, and Lawler J, 2006, Program on Technology Innovation: Advanced Light Source Research: ALITE-II: Losses in High Intensity Discharge Light Sources 1012994. (Palo Alto, CA: EPRI, Somerset, NJ: Philips Lighting Company, Niskayuna, NY: General Electric Company, Beverly, MA: Osram Sylvania) p 4-9.

[7] Smith D J, Bonvallet G A and Lawler J E, Infrared losses from a Na/Sc metal-halide high intensity discharge arc lamp. 2003, J. Phys. D: Appl. Phys. 36 p 1519-28

[8] Lawler J E, Koerber A and Weichmann U, Infrared continuum radiation from high and ultra-high pressure mercury lamps. 2005, J. Phys. D: Appl. Phys. 38 p 3071-85

[9] Herd M T and Lawler J E, Infrared continuum radiation from metal halide high intensity discharge lamps. 2007, J. Phys. D: Appl. Phys. 40 p 3386-95

[10] Bergman R S and Ohno Y, The Art and Science of Lamp Photometry. 2001, Procedings of the 9th International Symposium on the Science and Technology of Light Sources p 165-79

[11] Schafer R, Energy Efficient Light Sources. 1986, Proceedings 4th Int. Symp. on the Science and Technology of Light Sources, Karlsruhe, Germany p 52

[12] Kember D, Chenery D H, Sheppard N and Fell J, Fourier-transform i.r. emission studies of weakly emitting overlayers on metal surfaces. 1979, Spec. Acta. 35A p 455-9

[13] Chase D B, The Sensitivity and Limitations of Condensed Phase Infrared Emission Spectroscopy. 1981, App. Spec. 35-1 p 77-81

[14] Touloukian Y S and DeWitt D P, 1970, 'Thermophysical properties of matter part 7: Thermal radiative properties : metallic elements and alloys', New York: S.l. IFI/Plenum.

[15] Touloukian Y S, Powell R W and Ho C Y, 1970, 'Thermophysical properties of matter part 1: thermal conductivity: metallic elements and alloys' New York: S.l. IFI/Plenum.

[16] Kaye G W C, Laby T H, 1995, 'Tables of physical and chemical constants', 16th edition, Harlow: Longman,

[17] Rolling R E, Funai A I, and Grammer J R, 1964, Investigation of the effect of surface condition on the radiant properties of metals. TR No. AFML-TR-64-363. Air Force Systems Command, Wright-Patterson Air Force Base, Ohio.

[18] Private Communications, J van der Veen, J Suijker en M Haverlag, Philips Lighting Optical Calibration Laboratory (OCM) in Eindhoven 